\title{
SCIENTIFIC-PRACTICAL AND LEGAL PROBLEMS OF IMPLEMENTATION OF THE PERSONALIZED MEDICINE
}

\author{
N.O. Bezdieniezhnykh ${ }^{1, *}$, V.V. Reznikova ${ }^{2}$, O.V. Rossylna ${ }^{2}$ \\ ${ }^{1}$ R.E. Kavetsky Institute of Experimental Pathology, Oncology and Radiobiology, NAS of Ukraine, \\ Kyiv 03022, Ukraine \\ ${ }^{2}$ Taras Shevchenko National University of Kyiv, Kyiv 01033, Ukraine
}

The article is devoted to the comprehensive analysis of scientific, practical and legal issues of personalized medicine that is a rapidly developing science-driven approach to healthcare. It is concluded that there is lack of general legal framework for the encouragement of scientific researches and practical implementation in this field. The article shows foreign experience and prospects for the introduction of personalized medicine as a key concept of healthcare system, which is based on a selection of diagnostic, therapeutic and preventive measures that would be the most effective for a particular person in view of individual characteristics. The conclusions and proposals to improve the current legislation and development of personalized medicine in Ukraine are suggested. Key Words: personalized medicine, medical low, cancer treatment, cancer stem cells.

The beginning of the XXI century is marked by the completion of a number of global projects that give hope for substantial progress in the field of in the field of medical issues, including oncology. Thus, in particular, decoding of the genome, identification of genetic, epigenetic and transcriptional changes in normal and tumor cells brings scientists and doctors closer to new opportunities in early diagnosis and targeted treatment of cancer patients. Recently, a special hope is focused on the identification of normal and tumor stem cells that can be a modern platform in personalized approach for optimizing technology of diagnosis and treatment of patients. However, the successful development of the new vector of the medicine requires not only deep fundamental research and introduction of high technologies, but also parallel development and creation of the regulatory framework for their implementation.

The rapid pace of development the modern technologies requires the harmonization of scientificpractical aspects and existing problems of medical law, which will bring timely access to the real possibilities of personalized medicine. This means that activity (including economic) in the field of medical practice requires careful legislative regulation in order to provide the population with high-quality and affordable personalized medical assistance. Personalized medicine is such a model of health care based on a selection of diagnostic, therapeutic and preventive measures that would be best for a particular person in view of genetic, physiological, biochemical or other individual characteristics. Personalized medicine is a direction of modern medicine that uses individually oriented targeted diagnostic and therapeutic approaches. The essence of personalized medicine is reduced to the fact that a person, and not a disease, is placed at the center of the process of treatment and medical skill.

Submitted: August 4, 2017.

*Correspondence: E-mail: beznaliano@gmail.com

Abbreviation used: CSC - cancer stem cells.
Especially this mechanism is inherent in the treatment of patients with oncological diseases. This is due to various reasons and background of the disease, which is also accompanied by changes in different genes that are completely individual in each case.

The right of citizens to health care, unlike other human rights, has recently become legally enshrined in legal acts, including in the constitutions of the countries of the world community. Oddly enough, until the middle of the last century, in the constitutions of states all over the world the right of citizens to health was not mentioned, although many other human rights had already been proclaimed. Due to the prominent law reform in the health sector in many countries the subject of the role of medical practice is beginning to change in collaboration with other members of the medical and organizational processes. The implementation of legislative innovations implies structural and organizational changes in the health care system, the introduction of new organizational and financial mechanisms, ensuring economic independence, and increasing the diversity of organizational and legal forms of medical organizations.

In the policy of the Health 2020 program, the overall goals are a significant improvement in health and an increase in the welfare of the population, reduction of inequalities in health care, strengthening of public health and providing the availability of sustainable health systems tailored to individual needs of people. Such systems should be characterized by high quality of care and the principles of total coverage of medical care, social justice and sustainability. However, two main strategic objectives of the policy are to improve health for all people and to reduce inequalities with regard to health. Reducing inequities in health is achieved through the impact on the social and environmental determinants of health [1]. Albeit, despite the declared state's importance of implementing systemic and integrated health care reform, the Ministry of Health of Ukraine (the central executive body, which ensures the formation and implementation of state policy in the 
field of health care) works in this direction in extremely unsystematic way. For the successful implementation of the principles and mechanisms of the new European Health Policy 2020 in Ukraine, justification and development of a national strategy and program is necessary. It is advisable to direct the new national health policy in Ukraine to the implementation of strategic priorities in the field of preservation and strengthening of health, prevention of chronic non-infectious (especially oncological) diseases, minimization of the influence of risk factors of disease development on the basis of a cross-sectoral approach, creation of a favorable for health Environment, formation of responsible attitude of citizens to personal health and motivation of the population to a healthy lifestyle.

In such conditions, special attention should be focused on the issue of optimizing the organization and financing of the medical sector with the creation of an effective system of public health protection, through the priority development of primary health care, family medicine, personalized medicine, which are able to provide medical needs of the population at the level of current standards, taking into account the fundamentals of personalized medicine. After signing in 2014 of the Association Agreement with the European Union [2], electing Ukraine's European integration vector must ensure gradual forthcoming to European standards in the health care system through a reform aimed at creating a system focused on the patient, able to provide medical care for all Ukrainian citizens at the level of developed European states. The guideline for the reform was the European Union's “European Health Strategy 2020" program [3]. According to calculations of specialists, by 2020 the number of new cancer patients in Ukraine will exceed 200 thousand [4].

Our aim is to conduct a comprehensive analysis of theoretical, scientific and legal problems of implementation of personalized medicine in Ukraine.

According to Genomics and Personalized Medicine Act of 2010 the term "personalized medicine" means any clinical practice model that emphasizes the systematic use of preventive, diagnostic, and therapeutic interventions that use genome and family history information to improve health outcomes [5].

Although in recent years, there has been significant progress in clinical oncology: with the development of new antitumor drugs with targeted molecular action, the contingent of patients who receive effective specific therapy has improved their quality of life, but despite their success, cancer mortality remains high. At the same time, the empirical choice of the treatment regimen, the lack of selectivity and limited effectiveness of many antitumor drugs, a large number of side effects, as well as the high cost of therapy imply serious responsibility of the doctor. That is why the need for individualization of treatment becomes a topical issue in contemporary Ukraine, since knowledge of the molecular and genetic characteristics of the tumor and the patient will allow the personalization of thera- peutic regimens and reduce the risk of manifestations of toxicity of chemotherapeutic agents: an individual approach involves administering to a specific patient the required preparation in an adequate dose for certain indications in an effective period of time. Even with the rapid development of a new generation of anticancer drugs with targeted selective action - targeted drugs are designed to inhibit the growth, proliferation and viability of tumor cells through specific interactions with one or more target proteins. In this case, under the conditions of formally known molecular targets for each target drug, the response of the patient to treatment remains more individual and unpredictable. Therefore, the main task in oncology remains the choice of the most effective personalized treatment, which until now remains the mostly experimental.

"Personalized medicine is the dream of every oncologist and the legitimate expectation of every cancer patient", says Professor Martine Piccart, ESMO President. "However, currently we are not yet in the era of personalized oncology but in the era of stratified oncology, which means we are able to classify cancers according to critical targets against which we hope to develop effective drugs. Modern technologies such as deep DNA sequencing will be powerful tools in the future allowing us to identify drugable mutations" [6].

There is no official definition of personalized medicine in the European Union. But for the purpose of the European Commission report (31 October 2013) personalized medicine refers to a medical model using molecular profiling for tailoring the right therapeutic strategy for the right person at the right time, and/or to determine the predisposition to disease and/ or to deliver timely and targeted prevention [7].

America's legislation is more developed in this area. On May 27, 2010, in the House of Representatives, an A Bill with the aim to secure was adopted the promise of personalized medicine for all Americans by expanding and accelerating genomics research and initiatives to improve the accuracy of disease diagnosis, increase the safety of drugs, and identify novel treatments, and for other purposes.

Signed into law by the President on December $13^{\text {th }}$, 2016, the Act passed the House of Representatives (392-26) and Senate (94-5) with overwhelming bipartisan support. The Act secures $\$ 4.8$ billion in funding for the necessary infrastructure to advance precision medicine. This includes funding to the National Institutes of Health to support the President's Precision Medicine Initiative ( $\$ 1.5$ billion) and to Vice President Biden's "Cancer Moonshot" initiative ( $\$ 1.8$ billion). Spending under the Act will advance precision medicine more specifically by developing [8].

Can we find an analog of normative-legal regulation in the domestic legislation? Unlikely. Moreover, even none of the Concepts of the Ministry of Health does not foresee the movement of the health care system in Ukraine towards personalized medicine. Personalized medicine is a rapidly developing science-driven 
approach to health care. So does it need a specific legislation to regulate such social relationships?

Success in the treatment of cancer patients can be achieved in many respects only due to the successful combination of modern science-intensive technologies, regulated by legislation. However, unfortunately, deep knowledge of the nature of the malignant process is not always used in a timely manner in clinical practice because of the lack of necessary legal norms.

In what area should Ukrainian legislation be developed: medical or scientific and technical? Obviously, it will be impossible to restrict one normative legal act that would regulate the whole set of social relations in the field of personalized medicine, but there should be a key program document that will define the main concepts, principles, directions, and stages of the implementation of personalized medicine in Ukraine. In the future, the regulatory system should be formulated in a complex way, improving the current or developing a completely new legislation, whose fundamental task should be adequate protection: scientific development, medical secret, personal data, etc. The necessity of special regulatory regulation is also that in its absence it is impossible to realize scientific achievements in this sphere and to implement them in real medical practice, what is the urgent problem of the present.

Claiming to introduce the basics of personalized medicine, it is necessary to conduct in-depth analysis and refinement of the algorithm using a huge arsenal of basic and clinical knowledge. For the full implementation of the foundations of personalized medicine in Ukraine there is also a need for the proper regulation of corresponding social relations. Legislation should comprise and cover the following elements (components):

- An adequate level of protection of intellectual property rights for scientific developments, which are the driving force for the formation of personalized medicine. Medical diagnostics and genomic medicine are the key tools for many personalized therapies. In this regard, there are issues of intellectual property rights that need to be addressed. So, the world practice goes in such a way that the application for a patent for the diagnostic method should undergo even more careful analysis and verification. For example, in Mayo, the Supreme Court (the decision Mayo Collaborative Services v. Prometheus Labs., Inc.) determined that certain diagnostic analysis - such as a diagnostic test that correlates to a patient's health or disease - is not patentable when only the correlation between the diagnostic reading and the health status of the patient is the subject of the patent. The Supreme Court unanimously held that broadly claimed diagnostic methods are not patent-eligible because they attempt to patent a law of nature. As a result, patent applications claiming diagnostic methods are now more critically examined for patent-eligibility as well as novelty and non-obviousness [9].
In addition, the question arises about the ownership of genetic information.

- IT implementation, which will guarantee the security of personal data, the preservation of medical secrets, and the provision of high security standards for electronic health data. There is should be a balance between facilitating the exchange of medical records among health care providers and public health officials and the need to ensure the privacy of personal medical information.

- The foundation for protecting medical and genetic information, including a guarantee of non-discrimination based on the results of laboratory and other research, treatment, etc., including clinical and genetic research. For example, the Genetic Information Nondiscrimination Act of 2005 (GINA) [10] prohibits:

- medical insurance companies from discriminating against applicants on the basis of their genetic information;

- medical insurance companies from requesting that applicants for health coverage plans be genetically tested;

- employers from using genetic information to refuse employment and from collecting such data.

- A reliable mechanism for protecting the patient's rights to effective treatment with the corresponding responsibilities of the doctor to conduct appropriate diagnosis and therapy.

- Reform of state regulation of the health care system and elements of health insurance with maximum consideration of the above information, in particular the direction of the entire system for disease prevention and its diagnosis, which will significantly reduce the cost of treatment in the future, as well as stimulate and develop public-private partnerships.

According to expert estimates, about 250 variants of the tumor disease are differentiated based on only etiological, pathogenetic, organo- and tissue-specific features, symptomatic and prognostic course of the pathological process. In this case, the patient's response to the use of standard regimens and modes of administration of drugs, depending on the genetic constitution of the body, is accompanied by significant fluctuations in the effectiveness and safety of the therapy. For example, the frequency of development of unwanted drug reactions or even death in some countries goes to the $4-5^{\text {th }}$ place among all its causes. In addition, numerous pharmacoepidemiological studies indicate a complete lack of efficacy of drug therapy in $40 \%$ of patients. The individual variability of the body's reactions to medication can fluctuate within $20-95 \%$, the lack of response to pharmacotherapy $-10-40 \%$, the rate of excretion of drugs in different patients differs 4-40 times, the metabolism of drugs $-10-100$ times [11].

The factors that determine the response to the drug and affect the changes in the body's reaction are 
complex and function in combinations, some of which include the basic aspects of human biology, since a certain response to a drug directly affects the nature and life expectancy. Genetic variability is recognized as an important determinant of the individual variability of the drug response from clinical observations of the late 1950s (Kalow et al., 1957; Evans et al., 1960). Observing that the individual variability of the response to antitumor agents is often greater among the members of the population, compared to one person at different times, further emphasizes heredity as the main determinant of the response to the drug (Vesell, 1989; Kalow et al., 1998). These clinical and population data contributed to the formation of pharmacogenetics in the direction of assessing the genetic contribution to the individual variability in drug therapy [12].

Individual testing of tumor response in cancer treatment includes ex vivo tests that are important for predicting individual responses to the therapy. Such tests are developed on the basis of the response of the tumor cells to specific anticancer agents in vitro, which characterizes the sensitivity or resistance to a certain exogenous factor [13]. Previous studies have shown positive or negative aspects, peculiarities of application and limitation, and other properties and problems of such ex vivo analyzes. Indeed, there are a number of conceptual problems that do not depend on the chosen model for identifying an experimental model: an adequate choice of drug concentration relevant to the clinical situation; primary tumor or metastasis, heterogeneity of the tumor; the ability to maximally simulate the tumor microenvironment ex vivo. It is obvious that between the response of the patient to treatment and inhibition of tumor growth in vitro is a rather complex relationship [14], but despite this, numerous studies have achieved positive results for patients: more quick response to therapy, increasing longevity, reducing the number of chemotherapeutic treatment lines and side effects and reducing the cost of treatment [13]. Finally, some authors demonstrated $78.4 \%$ sensitivity and $90.1 \%$ specificity of individual tumor response testing $[13,15]$.

An important breakthrough in the sense of initiation and progression of cancer was the discovery of a cell subpopulation with features of stem cells, commonly referred to as cancer stem cells (CSC), a critical factor for a tumor. Most of the authors consider them responsible for the phenomenon of resistance to antitumor agents and disease progression $[16,17]$. Because of the presence of resistant CSC clones, each time in the treatment of the patient there is a variant of subsequent relapse of the tumor, which will be different, most likely, more resistant to therapy [18]. Undoubtedly, the choice of effective therapy is extremely important not only at the beginning of the treatment process, but also in the context of the progression of the disease, that is why the research on the most informative prognostic biomarkers, including markers, associated with the CSC phenotype, is extremely important. The targeting of anti-CSC therapy is a new paradigm in the treatment of cancer, which is one of the key tasks and areas of personalized medicine. Recently there was developed a new test that involves analyzing tumors for the CSC and parallel study of sensitivity to anticancer drugs ChemolD ${ }^{\circledR}$ [19-21]. Studies conducted using this test showed that patients with the same stage and other characteristics of the tumor process differ significantly in the clinical response to treatment, which also indicates the need for "personalization" in the diagnosis and treatment of cancer patients with a special approach to the identification and detail of tumor cells with CSC phenotype.

Thus, the concept of personalized medicine includes the use of clinical, genomic, transcriptional, proteomic and other information sources for the construction of an optimal algorithm for a person in terms of risk assessment, prevention and treatment: personalized medicine causes the rejection of the "protocol/standard" model of medicine characterized by empirical and "averaged" data [22]. In addition, understanding the "molecular portrait" of a person that causes specific properties in susceptibility to the disease, its progression and response to treatment will lead to the adaptation of medical care to the requirements of each individual patient [23].

By the time of the personalized medicine, the diagnosis of the tumor process, the prognosis of the course of the disease and the subsequent treatment were based on histological parameters, including the tissue origin and stage of differentiation. Experience has shown that morphological classification is often of little information and "scarce" in many aspects, since patients with the same histological diagnosis may have different responses to therapy and different course of the disease. Now we see the accumulation of vast amounts of data on molecular, genetic, epigenetic and other biomarkers of tumor development obtained by highly specific modern methods that are supported by new developments in computer systems analysis. Personalized medicine is a revolutionary concept that challenges the traditional fundamental and clinical oncology. Although the transition to personalized medicine requires a sufficiently large initial investment, in the long run such a direction will increase the profitability of future medical practice. The choice of cancer therapy today is usually based on prognostic factors, as well as on the experience and intuition of the doctor, but is associated with the risk of error in the treatment of a particular patient, which reduces the effectiveness of treatment, increases its cost and may even lead to tragic consequences. At the same time, the extremely significant disadvantage of modern antitumor therapy is the high cost of drugs, which also indicates the promise of a personified approach in the management of cancer patients, in terms of determining the sensitivity of the tumor of the patient to specific antitumor agents, thereby reducing the cost of treatment by a reasonable and adequate use of high-cost drugs. 
Personalized medicine has potentially significant benefits for patients, clinicians and health care systems alike. Some potential advantages offered by this new approach include:

- ability to make more informed medical decisions;

- higher probability of desired outcomes thanks to better-targeted therapies;

- reduced probability of adverse reactions to medicines;

- focus on prevention and prediction of disease rather than reaction to it;

- earlier disease intervention than has been possible in the past;

- improved health care cost containment [7].

Thus, today there is no definitive solution to the issues and problems for all types of cancer, but it becomes clear that personalized medicine is very important for patients and hospitals, and such "personalization" requires the humanization of medicine and begins with an individual doctor/patient relationship [6]. The perspective of the introduction of personalized medicine depends on the multidisciplinary approach: a team of doctors, experts, lawyers, legislators who, in teamwork and caring for the patient, will make realistic personalized medicine that will potentially improve the effectiveness of therapeutic strategies for cancer patients and increase their life expectancy to improve its quality. Although this poses a lot of problems in the organization of medical care, it is a necessary way to effectively treat each individual patient.

\section{REFERENCES}

1. Gruzieva TS. Innovative essence and strategic and practical potential of the new European policy «Health - 2020». Bull Problems Biol Med 2014; 1: 25-33.

2. Association Agreement between Ukraine, on the one hand, and the European Union, the European Atomic Energy Community and their Member States, on the other hand: International Document dated 27/06/2014. [Electronic resource]. http://zakon2.rada.gov.ua/laws/show/984_011.

3. Health 2020: the foundations of European politics in support of the actions of the whole state and society for the sake of health and well-being. WHO ERC, 2012. 18 p.

4. Prevention of oncological diseases. [Electronic resource]. https://www.unicef.org/ukraine/ukr/onko.pdf (in Ukrainian).

5. To secure the promise of personalized medicine for all Americans by expanding and accelerating genomics research and initiatives to improve the accuracy of disease diagnosis, increase the safety of drugs, and identify novel treatments, and for other purposes (Genomics and Personalized Medicine Act of 2010). A Bill 2010; H.R. 5440: 6. https://www. congress.gov/ 111/bills/hr5440/BILLS-111hr5440ih.pdf.
6. ESMO Patients Guide Series ESMO Personalised Medicine. $1^{\text {st }}$ ed. ESMO Press, 2013. 47 p.

7. European Commission. Use of '-omics' technologies in the development of personalised medicine. Commission staff working document 2013; 436: 5 .

8. Konski AF. Precision Medicine - Obama's Health Care Legacy. Personalized Med Bull 2016. https://www.personalizedmedicinebulletin.com/2016/12/ 18/3495/.

9. Konski AF. Personalized Medicine: Insights Into Current Legal Issues. Personalized Med Bull 2016. https://www.personalizedmedicinebulletin.com/2016/09/18/ personalized-medicine-insights-into-current-legal-issues/.

10. To prohibit discrimination on the basis of genetic information with respect to health insurance and employment. Genetic Information Nondiscrimination Act of 2005; S. 306: 6-75. https://www.congress.gov/bill/109th-congress/senate-bill/306/text.

11. Chekhun VF. From the systemic biology of cancer to the methodology of personalized treatment. Oncology 2012; 14: 84-8 (in Ukrainian).

12. Qiang M, Lu AYH. Pharmacogenetics, pharmacogenomics, and individualized medicine. Pharmacol Rev 2011; 63: $437-59$.

13. Giraud S, Loum E, Bessette B, et al. Oncogramme, a new promising method for individualized breast tumour response testing for cancer treatment. Anticancer Res 2011; 31: 139-46.

14. Samson DJ, Seidenfeld J, Ziegler K, Aronson N. Chemotherapy sensitivity and resistance assays: a systematic review. J Clin Oncol 2004; 22: 3618-30.

15. Nagourney RA. Ex vivo programmed cell death and the prediction of response to chemotherapy. Curr Treat Options Oncol 2006; 7: 103-10.

16. Sugihara E, Saya H. Complexity of cancer stem cells. Int J Cancer 2013; 132: 1249-59.

17. Colak S, Medema JP. Cancer stem cells - important players in tumor therapy resistance. FEBS J 2014; 281: 4779-91.

18. Howard CM, Valluri J, Claudio PP. The future of precision medicine in oncology: targeting cancer stem cells. Clinics Oncol 2017; 2: e1187.

19. Mathis SE, Alberico A, Nande R, et al. Chemopredictive assay for targeting cancer stem-like cells in patients affected by brain tumors. PLoS One 2014; 9: e105710.

20. Claudio PP, Mathis SE, Nande R, et al. ChemoID assay for glioblastoma. ASCO Ann Meet 2015; 33: e 13028.

21. Claudio PP, Mathis SE, Nande R, et al. Novel chemosensitivity assay for targeting cancer stem-like cells in brain tumors. ASCO Ann Meet 2014; 32: e13102.

22. Abrahams E, Silver M. The case for personalized medicine. J Diabetes Sci Technol 2009; 3: 680-4.

23. Ginsburg GS, Willard HF. Genomic and personalized medicine: foundations and applications. Transl Res 2009; 154: 277-87. 